\title{
Is right ventricular mid-septal pacing superior to apical pacing in patients with high degree atrio-ventricular block and moderately depressed left ventricular function?*
}

\author{
Kang $\mathrm{CHEN}^{1}$, Ye MAO ${ }^{2}$, Shao-hua LIU ${ }^{1}$, Qiong WU ${ }^{1}$, Qing-zhi LUO ${ }^{1}$, Wen-qi PAN ${ }^{1}$, Qi JIN ${ }^{1}$, Ning ZHANG ${ }^{1}$, \\ Tian-you LING ${ }^{1}$, Ying $\mathrm{CHEN}^{1}$, Gang GU ${ }^{1}$, Wei-feng SHEN ${ }^{\dagger+1}$, Li-qun WU ${ }^{\dagger+1}$ \\ ( ${ }^{1}$ Department of Cardiology, Ruijin Hospital, Shanghai Jiao Tong University School of Medicine, Shanghai 200025, China) \\ ( ${ }^{2}$ Outpatient Department, Shanghai Jiao Tong University School of Medicine, Shanghai 200025, China) \\ †E-mail: rjshenweifeng@gmail.com; wuliqun89@hotmail.com
}

Received Feb. 9, 2014; Revision accepted May 6, 2014; Crosschecked May 20, 2014

\begin{abstract}
Objective: We are aimed to investigate whether right ventricular mid-septal pacing (RVMSP) is superior to conventional right ventricular apical pacing (RVAP) in improving clinical functional capacity and left ventricular ejection fraction (LVEF) for patients with high-degree atrio-ventricular block and moderately depressed left ventricle (LV) function. Methods: Ninety-two patients with high-degree atrio-ventricular block and moderately reduced LVEF (ranging from $35 \%$ to $50 \%$ ) were randomly allocated to RVMSP $(n=45)$ and RVAP $(n=47)$. New York Heart Association (NYHA) functional class, echocardiographic LVEF, and distance during a 6-min walk test (6MWT) were determined at 18 months after pacemaker implantation. Serum levels of N-terminal pro-brain natriuretic peptide (NT-proBNP) were measured using an enzyme-linked immunosorbent assay (ELISA) kit. Results: Compared with baseline, NYHA functional class remained unchanged at 18 months, distance during 6MWT (485 m vs. $517 \mathrm{~m})$ and LVEF (36.7\% vs. 41.8\%) were increased, but BNP levels were reduced $(2352 \mathrm{pg} / \mathrm{ml}$ vs. $710 \mathrm{pg} / \mathrm{ml})$ in the RVMSP group compared with those in the RVAP group, especially in patients with LVEF $35 \%-40 \%$ (for all comparisons, $P<0.05$ ). However, clinical function capacity and LV function measurements were not significantly changed in patients with RVAP, despite the pacing measurements being similar in both groups, such as R-wave amplitude and capture threshold. Conclusions: RVMSP provides a better clinical utility, compared with RVAP, in patients with high-degree atrioventricular block and moderately depressed LV function whose LVEF levels ranged from $35 \%$ to $40 \%$.
\end{abstract}

Key words: Mid-septal pacing, Apical pacing, Impaired heart function doi: $10.1631 /$ jzus.B1400034

Document code: A

CLC number: $\mathrm{R} 541.7^{+} 6$

\section{Introduction}

Permanent cardiac pacing is the most efficient treatment for patients with high-degree atrioventricular (AV) block. Right ventricular apical pacing

\footnotetext{
Corresponding authors

* Project supported by the National Natural Science Foundation of China (Nos. 30900604 and 81270004), the Shanghai Natural Science Foundation (No. 11ZR1422800), the Medical Key Project of Shanghai Science and Technology Commission (No. 12411952100), and the New Outstanding Youth Program of Shanghai Municipal Commission of Health and Family Planning (No. XYQ2013105), China

(C) Zhejiang University and Springer-Verlag Berlin Heidelberg 2014
}

(RVAP) has been widely used for decades (Epstein et al., 2008), but artificial activation at the right ventricular apex usually alters the sequence of myocardial contraction and relaxation, resulting in abnormal septal wall motion and decreased ventricular function (Mera et al., 1999). This raises the serious issue that the right ventricular apex may be not an optimal pacing site, particularly for patients with reduced ventricular performance (Mera et al., 1999). In a review of 14 randomized studies, Shimony et al. (2012) found that right ventricular mid-septal pacing (RVMSP) is associated with a better left ventricular 
ejection fraction (LVEF) during follow-up, compared with RVAP. Nonetheless, previous studies mainly included patients with preserved LVEF (Victor et al., 1999; Stambler et al., 2003; Kypta et al., 2008; Gong et al., 2009; Zhang et al., 2012). Since patients with high-degree AV block and reduced cardiac function $(\mathrm{LVEF}<50 \%)$ often have rapid clinical deterioration and their life depends largely on a pacemaker with a physiological depolarization pattern, they represent optimal candidates to identify the effects of RVMSP (Victor et al., 1999; 2006; Stambler et al., 2003). Likewise, the optimal site to pace the right ventricle in patients who require frequent or continuous ventricular pacing owing to bradycardia but are not candidates for left ventricle (LV)-based cardiac resynchronization therapy, especially for those with impaired heart function, has not been clearly established.

The aim of the current study is to obtain more definitive evidence for the optimal site to pace the right ventricle and to identify suitable patients who could benefit from the optimized pacing site.

\section{Materials and methods}

\subsection{Study population}

A total of 272 patients with symptomatic II or III degree AV block, requiring elective permanent pacemaker implantation according to current guidelines (class I) from August 2008 to August 2011, were screened. We excluded patients with overt congestive heart failure and $\mathrm{LVEF}<35 \%(n=21)$, use of intravenous diuretics $3 \mathrm{~d}$ prior to implantation $(n=27)$, recent myocardial infarction ( $<3$ months) $(n=12)$, scheduled invasive cardiac procedure $(n=21)$, a history of atrial fibrillation requiring cardioversion $(n=18)$, suspected bi-nodal disease or sick sinus syndrome $(n=45)$, and clinically significant co-morbidity with an estimated life expectancy less than 1 year $(n=12)$, and patients unable to perform a 6-min walk test (6MWT) $(n=19)$. Among the 97 eligible patients, four who died of cardiac sudden death $(n=2)$ or malignancy $(n=2)$ and one who developed stroke during follow-up were also excluded because of the unavailability of LVEF and functional capacity assessment at 18 months. Thus, the remaining 92 patients (mean age $(73 \pm 13)$ years) were randomly allocated to permanent RVMSP or RVAP using a simple envelope drawing.
Conventional dual-chamber pacemakers without rate response functions including $\mathrm{C}^{50 \mathrm{D}^{\mathrm{TM}}}$ (Vitatron $^{\mathrm{TM}}$ Arnhem, the Netherlands), 5286 ${ }^{\mathrm{TM}}$ (St Jude ${ }^{\mathrm{TM}}$, Sylmar, CA, USA), or pacemaker with inactivated rate response function (DDD mode; Kappar ${ }^{\mathrm{TM}}$, Enpulse ${ }^{\mathrm{TM}}$, Adapt $^{\mathrm{TM}}$, Medtronic ${ }^{\mathrm{TM}}$, Minneapolis, MN, USA) were used in the study. Steroid eluting bipolar leads came from the same manufacturers $(1888 \mathrm{TC}, \mathrm{St}$ Jude $^{\mathrm{TM}}$, and Novus $5076^{\mathrm{TM}}$, Medtronic ${ }^{\mathrm{TM}}$ ). All programmable functions for reduction of ventricular pacing (e.g., AV hysteresis) had to be programmed 'off'. The AV delay was optimized based on echocardiographical images following the protocol described in the multicenter automatic defibrillator implantation trial-cardiac resynchronization therapy (MADIT-CRT) study after implantation (Goldenberg et al., 2011). The institutional ethics review board of the hospital approved the study and all subjects gave informed consent before randomization.

\subsection{Pacemaker implantation}

All procedures were performed under local anesthesia in an electrophysiology laboratory by two experienced electrophysiologists. The electrode leads were inserted through the right/left subclavian or cephalic veins. Standard passive leads were used for atrial implantation to locate the right atrial appendage. Patients randomized to the mid-septal position underwent implantation of an active fixation lead.

For RVMSP, the right ventricular mid-septal position was chosen with the aid of radiological anatomy using a standard technique, as shown in Fig. 1 (Lieberman et al., 2004; Kaye et al., 2009). In brief, under fluoroscopy at multiple projections (posteroanterior, right anterior oblique $10^{\circ}$, and left anterior oblique $40^{\circ}$ ) and with the help of electrocardiographic manifestations (a negative deflection in lead I and positive initial $\mathrm{R}$ waves of the paced ventricular complex in leads II and III (McGavigan et al., 2006)), the mid-septal position was precisely defined.

For RVAP, the conventional apical position of a passive-fixation ventricular lead required fluoroscopic superimposition of the tip of the lead with the diaphragm.

All measurements during pacemaker implantation were performed with the ERA 3000 analyzer (Biotronik, Germany). A pacing threshold of $<1.0 \mathrm{~V}$, an $\mathrm{R}$-wave of $>5.0 \mathrm{mV}$, and an impedance of 
500-1500 V were required for successful implantation. The P-wave had to be $>1.0 \mathrm{mV}$.

(a)

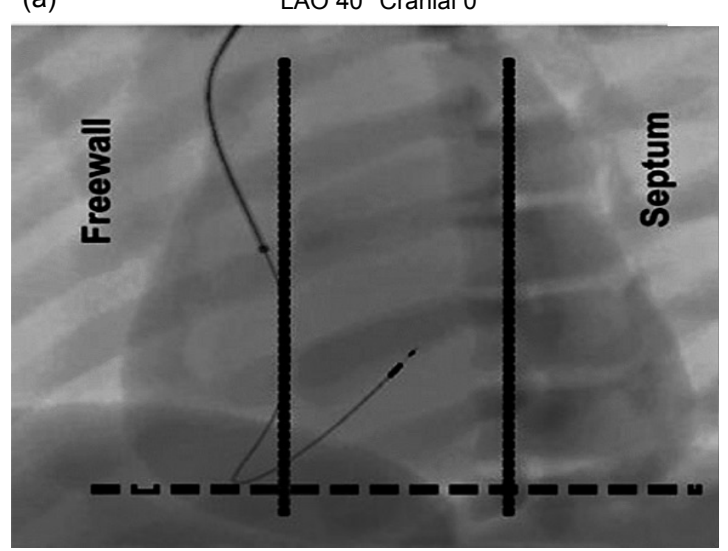

(b)

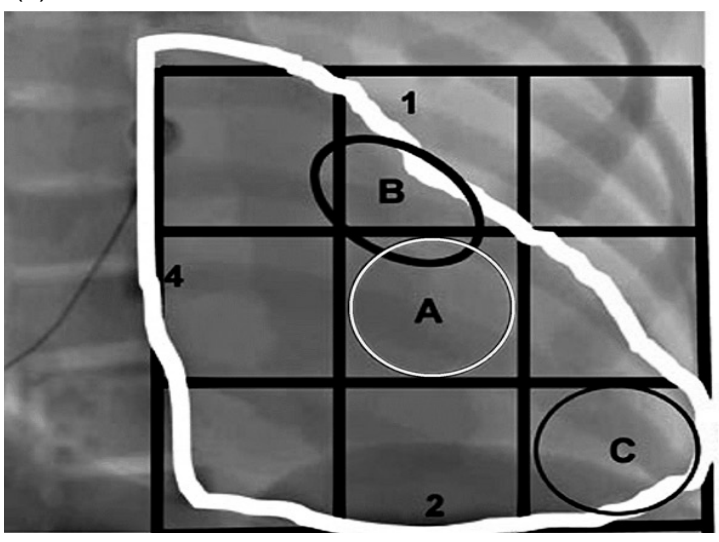

Fig. 1 Radiological anatomy of mid-septum access (a) $40^{\circ}$ left anterior oblique (LAO) fluoroscopy with the lead in septal position. The tip of the lead is geared for the column, in a direction opposite to the freewall of the right ventricle (RV). (b) Septal position is represented in the $10^{\circ}$ right anterior oblique (RAO) fluoroscopy. The letter A (white circle) represents the middle portion of the interventricular septum. The letter B (black circle) represents the high septal region and the letter $\mathrm{C}$ (black circle) the tip of RV. The figures are reproduced with permission from Lieberman et al. (2004)

\subsection{Pacemaker programming}

Initially, the pacemaker was programmed to DDD mode (60 beats $/ \mathrm{min})$ with an AV delay of $150 \mathrm{~ms}$. For measuring the paced QRS duration, a standard 12-lead surface electrocardiograph (ECG) at $25 \mathrm{~mm} / \mathrm{s}$ speed was recorded with a digital ECG writer (Marquette MAC-5000, GE, USA) and the QRS duration was automatically measured with the mean duration of all 12 leads. R-wave sensing, capture threshold, and lead impedance were measured immediately after pacemaker implantation and at each follow-up visit.

\subsection{Echocardiographic examination}

Transthoracic echocardiography was performed immediately after pacemaker implantation and at $7 \mathrm{~d}$ and 6 and 18 months during follow-up, respectively (Vivid 7, GE, USA). LV end-diastolic volume (EDV), end-systolic volume (ESV), and LVEF were determined by a modified Simpson's formula. The AV delay was set at a value that provides maximum separation of the E- and A-waves.

\subsection{MWT}

Distance during a symptom-limited 6MWT was recorded. During exercise, the pacemaker was programmed to a VDD mode with an upper limit pacing rate fixed at the maximum for each patient's age, and heart rate, cardiac rhythm, and blood pressure were continuously monitored.

\subsection{Follow-up}

Follow-up was completed in a special outpatient clinic or by telephone conversation with patients or their relatives. All data up to March 2013 were entered into a custom-built computerized database. The primary endpoint of the study was cardiac functional improvement from baseline to 18 months characterized by changes in plasma levels of $\mathrm{N}$-terminal pro-brain natriuretic peptide (NT-proBNP), LVEF, New York Heart Association (NYHA) class, and distance during 6MWT. Secondary endpoints consisted of the occurrence of overt congestive heart failure, mortality, and long-term safety of the alternative pacing site including stable values of lead impedance, R-wave, and pacing threshold.

\subsection{Statistical analysis}

All variables were summarized using frequency distributions for categorical variables and mean \pm standard deviation (SD) for continuous variables. Comparisons between groups (septal pacing or apical pacing) were performed using analysis of variance (ANOVA) for repeated measurement design data for continuous variables and $\chi^{2}$ test for categorical variables. Data were analyzed on an intention-to-treat basis. $P<0.05$ was considered statistically significant. 


\section{Results}

\subsection{Baseline characteristics}

Patients in RVMSP and RVAP groups did not differ significantly with respect to age, gender distribution, concomitant heart disease, medical treatments, and baseline LVEF. Seventeen patients in the RVMSP group and 15 in the RVAP group had LVEF ranging from $35 \%$ to $40 \%$ (Table 1 ).

\subsection{Pacing measurements}

There was no crossover from RVMSP to RVAP, and vice versa. In addition, the desired values for pacing threshold, R-wave amplitude, and resistance could be obtained in all patients. There was a trend towards shorter procedure duration and fluoroscopy time $[(52 \pm 6)$ vs. $(62 \pm 4) \mathrm{min}, P<0.05 ;(5.7 \pm 3)$ vs. (6.3 \pm 2 ) $\min , P=0.277]$ for RVMSP. No complications or lead dislodgement occurred during the study period.

In patients with lower LVEF (35\%-40\%), RVMSP was associated with significantly shorter QRS and lower lead impedance than RVAP, whereas R-wave and capture threshold remained stable (Table 2). No clinical lead failure was observed.

\subsection{Follow-up outcomes}

During 18 months of follow-up, persistent or permanent atrial fibrillation occurred in one patient of the RVMSP group and in two of the RVAP group. Serum BNP levels, LVEF, and distance during the 6MWT were not significantly changed for patients with high LV function (LVEF 40\%-50\%) in both
Table 1 Clinical characteristics of patients in RVMSP and RVAP groups

\begin{tabular}{|c|c|c|c|}
\hline \multirow[b]{2}{*}{ Parameter } & \multicolumn{2}{|c|}{ Value $^{*}$} & \multirow[b]{2}{*}{$P$} \\
\hline & $\begin{array}{c}\text { RVMSP } \\
(n=45)\end{array}$ & $\begin{array}{l}\text { RVAP } \\
(n=47)\end{array}$ & \\
\hline Age (year) & $70 \pm 15$ & $75 \pm 10$ & NS \\
\hline Female & $22(49 \%)$ & $15(32 \%)$ & NS \\
\hline The third degree AV-block & 25 & 26 & NS \\
\hline Narrow QRS & $10(22 \%)$ & $11(23 \%)$ & NS \\
\hline Wide QRS & $15(33 \%)$ & $15(32 \%)$ & NS \\
\hline Left bundle branch block & $9(20 \%)$ & $8(17 \%)$ & NS \\
\hline Right bundle branch block & $6(13 \%)$ & $7(15 \%)$ & NS \\
\hline The second degree AV-block & 20 & 21 & NS \\
\hline Narrow QRS & $10(22 \%)$ & $11(23 \%)$ & NS \\
\hline Wide QRS & $10(22 \%)$ & $10(21 \%)$ & NS \\
\hline Left bundle branch block & $6(13 \%)$ & $5(11 \%)$ & NS \\
\hline Right bundle branch block & $4(9 \%)$ & $5(10 \%)$ & NS \\
\hline Coronary heart disease & $14(31 \%)$ & $19(40 \%)$ & NS \\
\hline Hypertension & $22(49 \%)$ & $24(51 \%)$ & NS \\
\hline Diabetes & $13(29 \%)$ & $10(21 \%)$ & NS \\
\hline Hyperlipidemia & $11(24 \%)$ & $13(28 \%)$ & NS \\
\hline $35 \%<\mathrm{LVEF} \leq 40 \%$ & $17(38 \%)$ & $15(32 \%)$ & NS \\
\hline $40 \%<\mathrm{LVEF} \leq 50 \%$ & $28(62 \%)$ & $32(68 \%)$ & NS \\
\hline \multicolumn{4}{|l|}{ Pharmacologic therapy } \\
\hline $\begin{array}{l}\text { ACE inhibitor or AT II } \\
\text { blocker }\end{array}$ & $27(60 \%)$ & $31(69 \%)$ & NS \\
\hline$\beta$-Blocker & $8(18 \%)$ & $7(15 \%)$ & NS \\
\hline Loop diuretic & $6(13 \%)$ & $9(19 \%)$ & NS \\
\hline Calcium channel blocker & $2(4 \%)$ & $3(6 \%)$ & NS \\
\hline
\end{tabular}

Table 2 Pacing measurements at baseline and after 18 months according to LVEF level

\begin{tabular}{|c|c|c|c|c|c|c|c|c|c|c|c|}
\hline \multicolumn{2}{|c|}{ Group } & $\begin{array}{l}\text { R-wave } \\
\text { amplitude } \\
\text { (V) }\end{array}$ & $P$ & $\begin{array}{c}\text { Capture } \\
\text { threshold } \\
(\mathrm{mV})\end{array}$ & $P$ & $\begin{array}{c}\text { Lead } \\
\text { impendence } \\
(\Omega) \\
\end{array}$ & $P$ & $\begin{array}{c}\text { QRS } \\
\text { duration } \\
(\mathrm{ms})\end{array}$ & $P$ & $\begin{array}{l}\text { Ventricular } \\
\text { pacing }(\%)\end{array}$ & $P$ \\
\hline \multicolumn{12}{|c|}{ Low LVEF $(35 \%-40 \%)$} \\
\hline \multirow[t]{2}{*}{ Baseline } & Septal & $11.0 \pm 3.5$ & 0.398 & $0.7 \pm 0.2$ & 0.162 & $778 \pm 211$ & 0.041 & $144 \pm 23$ & 0.023 & & \\
\hline & Apical & $12.7 \pm 6.9$ & & $0.6 \pm 0.2$ & & $913 \pm 130$ & & $165 \pm 26$ & & & \\
\hline \multirow[t]{2}{*}{18 months } & Septal & $14.4 \pm 7.9$ & 0.165 & $0.7 \pm 0.3$ & 0.754 & $566 \pm 201$ & 0.019 & $134 \pm 32$ & $<0.001$ & $91 \pm 20$ & 0.875 \\
\hline & Apical & $11.2 \pm 4.6$ & & $0.7 \pm 0.4$ & & $732 \pm 170$ & & $173 \pm 22$ & & $95 \pm 17$ & \\
\hline \multicolumn{12}{|c|}{ High LVEF (40\%-50\%) } \\
\hline \multirow[t]{2}{*}{ Baseline } & Septal & $11.5 \pm 5.7$ & 0.381 & $0.7 \pm 0.3$ & 0.417 & $680 \pm 96$ & 0.002 & $149 \pm 23$ & 0.022 & & \\
\hline & Apical & $12.7 \pm 4.5$ & & $0.6 \pm 0.2$ & & $807 \pm 192$ & & $164 \pm 25$ & & & \\
\hline \multirow[t]{2}{*}{18 months } & Septal & $12.3 \pm 5.4$ & 0.408 & $0.7 \pm 0.2$ & 0.538 & $610 \pm 120$ & 0.013 & $153 \pm 18$ & $<0.001$ & $93 \pm 16$ & 0.925 \\
\hline & Apical & $11.3 \pm 3.7$ & & $0.7 \pm 0.4$ & & $711 \pm 175$ & & $175 \pm 20$ & & $94 \pm 21$ & \\
\hline
\end{tabular}

Data are expressed as mean \pm SD 
RVMSP and RVAP groups. However, patients with poor LV function (LVEF 35\%-40\%) had better clinical results after RVMSP compared with those with RVAP (Fig. 2). After 18 months of follow-up, the patients allocated to the RVMSP group with poor LV function (LVEF 35\%-40\%) had lower serum BNP levels [(710.71 \pm 682.73$)$ vs. $(2352.13 \pm 1775.00) \mathrm{pg} / \mathrm{ml}$, $P<0.05]$, higher LVEF levels [(41.8 \pm 2.2$) \%$ vs. $(36.7 \pm$ $0.7) \%, P<0.05]$, and improved exercise tolerance [6MWT: $(516.6 \pm 37.3)$ vs. $(485.9 \pm 25.4) \mathrm{m}, P<0.05$ ] (Fig. 2). Interestingly, even in the same septal-pacing condition, the lower LVEF group had lower serum BNP levels compared with the high LV function group [(710.71 \pm 682.73$)$ vs. $(1776.46 \pm 1869.71) \mathrm{pg} / \mathrm{ml}$, $P<0.05]$ (Fig. 3), which indicates that patients with moderately depressed LV function could obtain more clinical benefit from septal-pacing.

\section{Discussion}

Our study is the first to indicate that RVMSP provides a better clinical utility, compared with RVAP, in patients with high-degree AV-block and moderately depressed LV function whose LVEF levels range from $35 \%$ to $40 \%$.

Compared with normal intrinsic conduction through the His-Purkinje system, RVAP is associated with abnormal electrical activation and contraction sequence, systolic and diastolic dysfunction, perfusion mismatch, increased energy expenditure, and histological abnormalities (Lee et al., 1994; Tantengco et al., 2001). To date, whether an optimal right ventricular site or sites for long-term permanent pacing exist has not been determined, especially in patients with impaired cardiac function (LVEF ranging from $35 \%$ to $40 \%$ ).

Most previous studies focused on patients with normal cardiac function and reported no statistically significant difference in clinical outcomes between apical pacing and septal pacing (Flevari et al., 2009; Cano et al., 2010; Leong et al., 2010). In the present single-centre study comparing the long-term effects of standard RVAP vs. RVMSP, we chose AV block patients requiring continuous pacing with reduced LV function (LVEF 35\%-50\%). Although these patients are not the candidates for LV-based

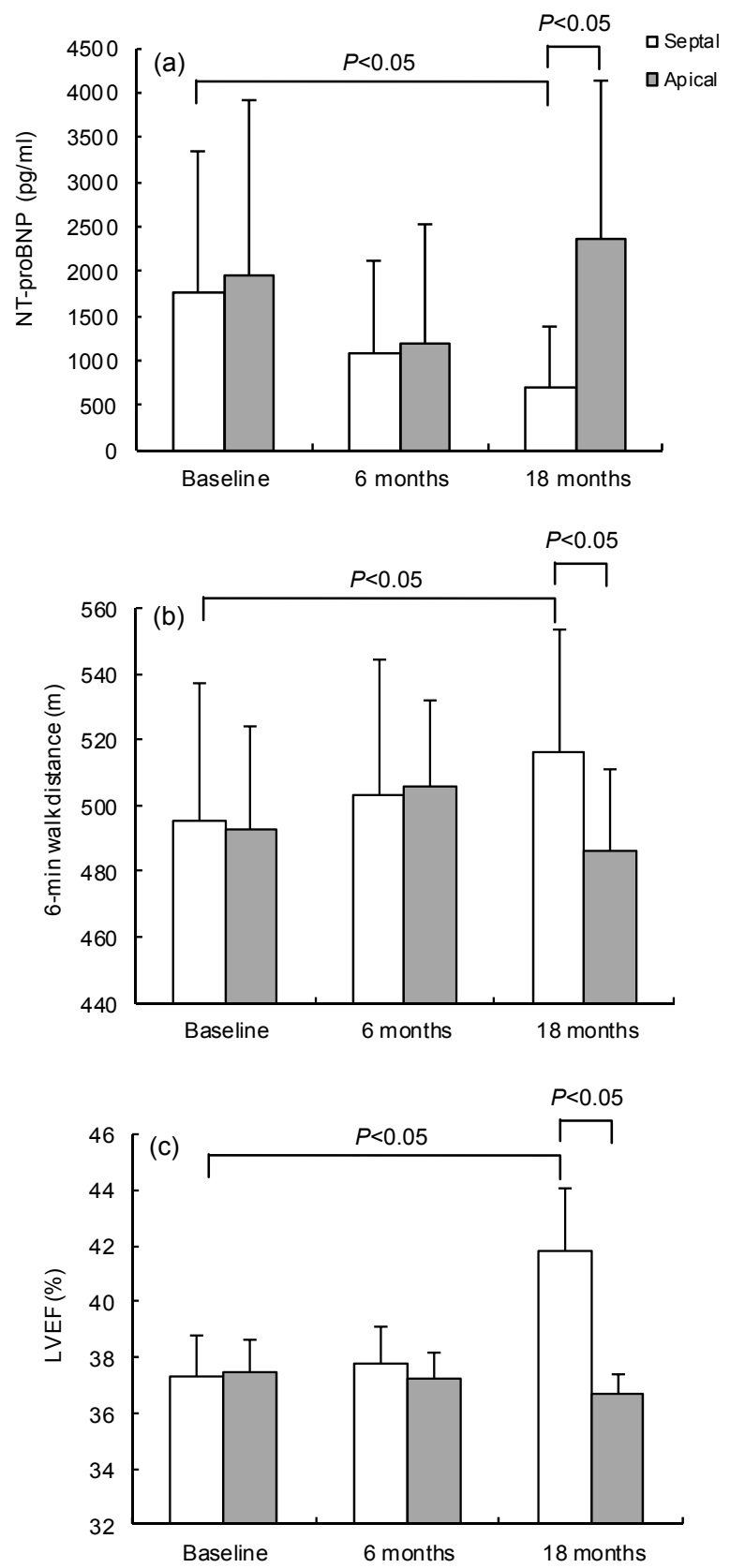

Fig. 2 BNP (a), 6MWT (b), and LVEF (c) at baseline and during follow-up in patients with $35 \%<\mathrm{LVEF} \leq \mathbf{4 0 \%}$

(a) Patients randomized to the RVMSP group with $35 \%<$ $\mathrm{LVEF} \leq 40 \%$ at baseline have an obvious BNP which is decreasing during follow-up compared with those in the RVAP group $(P<0.05)$; (b) 6WMT shows a significant increase in patients with $35 \%<\mathrm{LVEF} \leq 40 \%$ allocated to the RVMSP group compared with the RVAP group after 18 months' follow-up $(P<0.05)$; (c) After 18 months' follow-up, patients with $35 \%<\mathrm{LVEF} \leq 40 \%$ at baseline allocated to RVMSP have a significant increase compared with $\operatorname{RVAP}(P<0.05)$. Data are expressed as mean $\pm \mathrm{SD}$ 


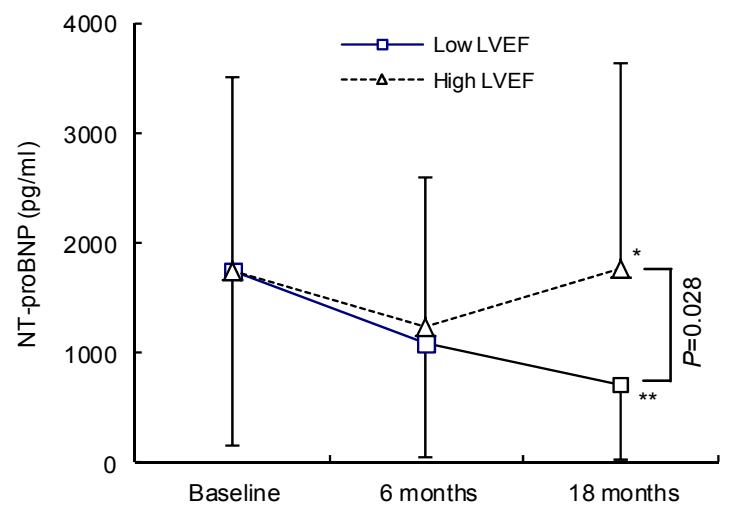

Fig. 3 BNP at low and high LVEF groups within mid-septal pacing

Different levels of LVEF appear to be a key factor for the therapy of mid-septal pacing. The lower LVEF $(35 \%<$ $\mathrm{LVEF} \leq 40 \%$ ) group has a better BNP-decreasing effect than the higher LVEF $(40 \%<\mathrm{LVEF} \leq 50 \%)$ group based on the same mid-septal pacing conditions $(P<0.05)$. ${ }^{*} P>0.05$, ${ }^{* *} P<0.05$, vs. baseline. Data are expressed as mean \pm SD

cardiac resynchronization treatment (CRT) according to the American College of Cardiology/American Heart Association/Heart Rhythm Society guidelines, they might still need the optimal cardiac pace which could improve heart function. Also, most patients find the cost of CRT to be too high.

In this study, we have demonstrated that pacing of different right ventricular areas affects cardiac function in patients with lower LVEF over a long-term follow-up. At 18 months, LVEF was increased in patients with lower baseline values (35\%-40\%) after RVMSP compared with those after RVAP, coincident with an increase in distance during 6MWT and a decrease in serum BNP level, which are routinely used to evaluate cardiac function and hemodynamic status in clinical practice. In contrast, no statistically significant difference was detected in clinical outcomes for patients with relatively preserved left ventricular function (LVEF $40 \%-50 \%$ ), irrespective of right ventricular pacing site. These findings support the notion (Flevari et al., 2009; Cano et al., 2010; Leong et al., 2010) that protection or restoration of ventricular synchrony is critical for patients with reduced LV systolic function (Cleland $e t$ al., 2005; Doshi et al., 2005; Kindermann et al., 2006; Muto et al., 2007), but may be of less importance for those with normal LV systolic function. Victor et al.
(2006) found that in contrast to RVAP, RVMSP preserved LVEF in patients with a baseline value of $\leq 45 \%$, but did not improve LVEF in patients with baseline LVEF $>45 \%$. Sweeney and Hellkamp (2006) showed that in patients with normal LV systolic function without myocardial infarction, the risk of heart failure after RVAP was low and RVMSP may provide no benefit over RVAP for these patients (Chiladakis et al., 2007). Stambler et al. (2003) conducted a randomized, crossover trial to determine whether quality of life was better after 3 months of right ventricular outflow tract (RVOT) pacing than after RVAP in patients with chronic heart failure and LVEF $\leq 40 \%$. They found RVOT pacing had no clinical advantage over RVAP. However, in their study,

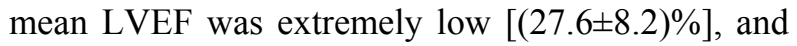
severely depressed cardiac function is generally irreversible. CRT should be recommended for these patients. In contrast, non-physiological pacing exerts a deleterious effect in patients with reduced LVEF $(35 \%-40 \%)$. Based on the results, we would choose patients with lower LVEF (35\%-40\%) as the optimal candidates for RVMSP.

Interestingly, in the present study, no difference in chronic thresholds was found between RVAP and RVMSP after a mean follow-up of 1.5 years. This is consistent with previous findings (Vlay, 2006). We used screw-in leads and all implantations were successful.

While a difference in LVEF at 18 months was detected between various pacing sites, the results should be interpreted with caution given the observed effect size. The small pooled differences fall within the inter-observer and intra-observer variabilities for LVEF measurement (Vlay, 2006). Finally, the optimal pacing site at the septum (high, mid, or low) requires further investigation.

\section{Conclusions}

This study confirmed the reliability and safety of long-term RVMSP with screw-in leads. Compared with RVAP, RVMSP results in significant improvements in cardiac function or LVEF at 18 months, particularly for patients with lower LVEF $(35 \%-40 \%)$. Larger randomized trials are needed to confirm the role of LVEF levels in optimizing the pacing site. 


\section{Acknowledgements}

We thank Dr. Randy A. LIEBERMAN (Division of Cardiology and Electrophysiology, Harper Hospital, Detroit, Michigan, USA) for his help in sharing the fluoroscopic figures.

\section{Compliance with ethics guidelines}

Kang CHEN, Ye MAO, Shao-hua LIU, Qiong WU, Qing-zhi LUO, Wen-qi PAN, Qi JIN, Ning ZHANG, Tian-you LING, Ying CHEN, Gang GU, Wei-feng SHEN, and Li-qun WU declare that they have no conflict of interest.

All procedures followed were in accordance with the ethical standards of the responsible committee on human experimentation (institutional and national) and with the Helsinki Declaration of 1975, as revised in 2008 (5). Informed consent was obtained from all patients for being included in the study. Additional informed consent was obtained from all patients for which identifying information is included in this article.

\section{References}

Cano, O., Osca, J., Sancho-Tello, M.J., et al., 2010. Comparison of effectiveness of right ventricular septal pacing versus right ventricular apical pacing. Am. J. Cardiol., 105(10): 1426-1432. [doi:10.1016/j.amjcard.2010.01.004]

Chiladakis, J.A., Koutsogiannis, N., Kalogeropoulos, A., et al., 2007. Permanent and atrial-synchronized ventricular stimulation for clinically stable patients with normal or impaired left ventricular systolic function. Pacing Clin. Electrophysiol., 30(2):182-187. [doi:10.1111/j.1540-8159. 2007.00647.x]

Cleland, J.G., Daubert, J.C., Erdmann, E., et al., 2005. The effect of cardiac resynchronization on morbidity and mortality in heart failure. N. Engl. J. Med., 352(15): 1539-1549. [doi:10.1056/NEJMoa050496]

Doshi, R.N., Daoud, E.G., Fellows, C., et al., 2005. Left ventricular-based cardiac stimulation post AV nodal ablation evaluation (the pave study). J. Cardiovasc. Electrophysiol., 16(11):1160-1165. [doi:10.1111/j.1540-8167. 2005.50062.x]

Epstein, A.E., Dimarco, J.P., Ellenbogen, K.A., et al., 2008. ACC/AHA/HRS 2008 guidelines for device-based therapy of cardiac rhythm abnormalities. Heart Rhythm., 5(6): e1-e62. [doi:10.1016/j.hrthm.2008.04.014]

Flevari, P., Leftheriotis, D., Fountoulaki, K., et al., 2009. Long-term nonoutflow septal versus apical right ventricular pacing: relation to left ventricular dyssynchrony. Pacing Clin. Electrophysiol., 32(3):354-362. [doi:10. 1111/j.1540-8159.2008.02244.x]

Goldenberg, I., Moss, A.J., Hall, W.J., et al., 2011. Predictors of response to cardiac resynchronization therapy in the multicenter automatic defibrillator implantation trial with cardiac resynchronization therapy (MADIT-CRT). Circulation, 124(14):1527-1536. [doi:10.1161/CIRCULATIONAHA. $110.014324]$

Gong, X., Su, Y., Pan, W., et al., 2009. Is right ventricular outflow tract pacing superior to right ventricular apex pacing in patients with normal cardiac function? Clin. Cardiol., 32(12):695-699. [doi:10.1002/clc.20677]

Kaye, G., Stambler, B.S., Yee, R., 2009. Search for the optimal right ventricular pacing site: design and implementation of three randomized multicenter clinical trials. Pacing Clin. Electrophysiol., 32(4):426-433. [doi:10.1111/j. 1540-8159.2009.02301.x]

Kindermann, M., Hennen, B., Jung, J., et al., 2006. Biventricular versus conventional right ventricular stimulation for patients with standard pacing indication and left ventricular dysfunction: the Homburg Biventricular Pacing Evaluation (HOBIPACE). J. Am. Coll. Cardiol., 47(10): 1927-1937. [doi:10.1016/j.jacc.2005.12.056]

Kypta, A., Steinwender, C., Kammler, J., et al., 2008. Long-term outcomes in patients with atrioventricular block undergoing septal ventricular lead implantation compared with standard apical pacing. Europace, 10(5): 574-579. [doi:10.1093/europace/eun085]

Lee, M.A., Dae, M.W., Langberg, J.J., et al., 1994. Effects of long-term right ventricular apical pacing on left ventricular perfusion, innervation, function and histology. J. Am. Coll. Cardiol., 24(1):225-232. [doi:10.1016/0735-1097 (94)90567-3]

Leong, D.P., Mitchell, A.M., Salna, I., et al., 2010. Long-term mechanical consequences of permanent right ventricular pacing: effect of pacing site. J. Cardiovasc. Electrophysiol., 21(10):1120-1126. [doi:10.1111/j.1540-8167.2010.01804.x]

Lieberman, R., Grenz, D., Mond, H.G., et al., 2004. Selective site pacing: defining and reaching the selected site. Pacing Clin. Electrophysiol., 27(6 Pt 2):883-886. [doi:10. 1111/j.1540-8159.2004.00551.x]

McGavigan, A.D., Roberts-Thomson, K.C., Hillock, R.J., et al., 2006. Right ventricular outflow tract pacing: radiographic and electrocardiographic correlates of lead position. Pacing Clin. Electrophysiol., 29(10):1063-1068. [doi:10.1111/j.1540-8159.2006.00499.x]

Mera, F., Delurgio, D.B., Patterson, R.E., et al., 1999. A comparison of ventricular function during high right ventricular septal and apical pacing after His-bundle ablation for refractory atrial fibrillation. Pacing Clin. Electrophysiol., 22(8):1234-1239. [doi:10.1111/j.1540-8159. 1999.tb00606.x]

Muto, C., Ottaviano, L., Canciello, M., et al., 2007. Effect of pacing the right ventricular mid-septum tract in patients with permanent atrial fibrillation and low ejection fraction. J. Cardiovasc. Electrophysiol., 18(10):1032-1036. [doi:10.1111/j.1540-8167.2007.00914.x]

Shimony, A., Eisenberg, M.J., Filion, K.B., et al., 2012. Beneficial effects of right ventricular non-apical vs. apical pacing: a systematic review and meta-analysis of randomized-controlled trials. Europace, 14(1):81-91. [doi:10.1093/europace/eur240]

Stambler, B.S., Ellenbogen, K., Zhang, X., et al., 2003. Right ventricular outflow versus apical pacing in pacemaker patients with congestive heart failure and atrial fibrillation. 
J. Cardiovasc. Electrophysiol., 14(11):1180-1186. [doi: 10.1046/j.1540-8167.2003.03216.x]

Sweeney, M.O., Hellkamp, A.S., 2006. Heart failure during cardiac pacing. Circulation, 113(17):2082-2088. [doi:10. 1161/CIRCULATIONAHA.105.608356]

Tantengco, M.V., Thomas, R.L., Karpawich, P.P., 2001. Left ventricular dysfunction after long-term right ventricular apical pacing in the young. J. Am. Coll. Cardiol., 37(8): 2093-2100. [doi:10.1016/S0735-1097(01)01302-X]

Victor, F., Leclercq, C., Mabo, P., et al., 1999. Optimal right ventricular pacing site in chronically implanted patients: a prospective randomized crossover comparison of apical and outflow tract pacing. J. Am. Coll. Cardiol., 33(2): 311-316. [doi:10.1016/S0735-1097(98)00589-0]
Victor, F., Mabo, P., Mansour, H., et al., 2006. A randomized comparison of permanent septal versus apical right ventricular pacing: short-term results. J. Cardiovasc. Electrophysiol., 17(3):238-242. [doi:10.1111/j.1540-8167. 2006.00358.x]

Vlay, S.C., 2006. Right ventricular outflow tract pacing: practical and beneficial. A 9-year experience of 460 consecutive implants. Pacing Clin. Electrophysiol., 29(10): 1055-1062. [doi:10.1111/j.1540-8159.2006.00498.x]

Zhang, H.X., Qian, J., Hou, F.Q., et al., 2012. Comparison of right ventricular apex and right ventricular outflow tract septum pacing in the elderly with normal left ventricular ejection fraction: long-term follow-up. Kardiol. Pol., 70(11):1130-1139.

\section{中文栍要：}

\section{本文题目：轻度心功能受损的高度房室传导阻滞患者采用中位间隔部起搏是否优于心尖部起搏?}

Is right ventricular mid-septal pacing superior to apical pacing in patients with high degree atrio-ventricular block and moderately depressed left ventricular function?

研究目的: 对于轻度心功能受损的高度房室传导阻滞患者, 明确右室起搏的最优化位置, 同时明确哪一 类患者能够从最优化的右心室起搏中获益。

创新要点: 首次明确相比于右心室心尖部起搏, 中位间隔部起搏明显改善轻度心功能受损, 尤其是左室 射血分数在 35\% 40\%的高度房室传导阻滞患者的临床预后。

研究方法: 随机分配进入中位间隔部起搏或心尖部起搏的患者, 在基线状态下记录 $\mathrm{N}$-末端脑钠肽前体、 六分钟步行试验以及心超结果; 随访 18 个月后观察上述结果变化。同时, 根据射血分数的 不同进行亚组分析。

重要结论: 相比于右心室心尖部起搏, 中位间隔部起搏能够改善轻度心功能受损的高度房室传导阻滞患 者的临床预后，尤其对于左室射血分数在 35\% 40\%的患者。

关键词组：右心室中位间隔部起搏；右心室心尖部起搏；心功能受损 